\title{
Effect of Fuzheng Huayu capsule combined with Pegasys on genotype 1 hepatitis $\mathbf{C}$ fibrosis and cell apoptosis
}

\author{
BO ZHANG, MINTAO HU, LIHUA HUANG, YUNCHUAN PU, HAO PEI, ZHONG HUA and SHANGZHI YAO
}

Department of Infectious Diseases, The Fifth People's Hospital of Wuxi, Wuxi, Jiangsu 214005, P.R. China

Received February 4, 2014; Accepted July 28, 2014

DOI: $10.3892 /$ etm.2014.1891

\begin{abstract}
The aim of the present study was to observe the effects of Fuzheng Huayu capsule combined with Pegasys (peginterferon $\alpha-2 a$ ) on hepatic fibrosis in rats and in the treatment of patients with genotype 1 hepatitis $C$ and hepatic cirrhosis. A dimethylnitrosamine (DMN)-induced rat model of liver injury was established. Fuzheng Huayu capsule combined with Pegasys was administered to the rats and the DMN-induced hepatocyte apoptosis was observed. In addition, a total of 100 patients with genotype 1 hepatitis $\mathrm{C}$ and hepatic cirrhosis were treated by oral administration of Fuzheng Huayu capsule combined with Pegasys or with Pegasys alone. The therapeutic effect of Fuzheng Huayu capsule combined with Pegasys was analyzed. Following the oral administration of Fuzheng Huayu capsule combined with Pegasys to the DMN model rats, the expression of $\alpha$-smooth muscle actin was found to be significantly reduced, hemopoietic stem cell apoptosis was increased and liver cell apoptosis was reduced. These indices were significantly different compared with those in the model group $(\mathrm{P}<0.05)$. Liver function and liver fibrosis were markedly recovered in hepatitis $\mathrm{C}$ patients with hepatic cirrhosis following treatment with the combination treatment compared with those in the patients treated with Pegasys alone $(\mathrm{P}<0.05)$. In conclusion, the combination of Fuzheng Huayu capsule with Pegasys inhibited liver fibrosis and cell apoptosis, and may be a novel therapeutic strategy for the treatment of patients with compensated cirrhosis due to hepatitis C. This study provides a method for the optimization of existing treatment strategies and for the establishment of potentially effective combination therapies.
\end{abstract}

\section{Introduction}

Hepatitis $\mathrm{C}$ is an infectious disease that is prevalent around the world. Hepatitis $\mathrm{C}$ virus (HCV) induces chronic infec-

Correspondence to: Dr Shangzhi Yao, Department of Infectious Diseases, The Fifth People's Hospital of Wuxi, 88 Xingyuan Road, Wuxi, Jiangsu 214005, P.R. China

E-mail: shangzhiyaocn@163.com

Key words: Fuzheng Huayu capsule, Pegasys, rat model, liver fibrosis tion in $\sim 80 \%$ of infected individuals. Approximately $10-20 \%$ of patients develop cirrhosis and nearly $5 \%$ will eventually suffer from liver cancer (1). The aim of HCV treatment is to clear the virus and the symptoms associated with hepatitis $\mathrm{C}$ infection $(2,3)$. At present, combined treatment with pegylated interferon $\alpha$ (PEG-IFN) and the antiviral drug ribavirin is the standard approach for HCV treatment (4-6). In a previous study, the sustained virological response (SVR) was found to be 8,15 and $30 \%$ in patients with chronic hepatitis $\mathrm{C}$ and cirrhosis following treatment with IFN, $90 \mu \mathrm{g}$ PEG-IFN and $180 \mu \mathrm{g}$ PEG-IFN, respectively (7). In the present study, the effect of Fuzheng Huayu capsule combined with Pegasys (peginterferon $\alpha-2 a$ ) was investigated on cell apoptosis in rats with dimethylnitrosamine (DMN)-induced liver injury and on liver function and fibrosis in patients with hepatitis $\mathrm{C}$ and hepatic cirrhosis. These results may provide a novel therapeutic strategy for the treatment of patients with hepatic cirrhosis.

\section{Materials and methods}

Animals. Atotal of 60 Wistar male rats $(200 \pm 10 \mathrm{~g})$ were provided by Jiangsu Laboratory Animal Center (Suzhou, China). This study was performed in accordance with the recommendations in the Guide for the Care and Use of Laboratory Animals of the National Institutes of Health (8th edition, Bethesda, MD, USA, 2010). The animal use protocol was reviewed and approved by the Institutional Animal Care and Use Committee of the Fifth People's Hospital of Wuxi (Wuxi, China). The rats were randomly divided into two groups, a normal control group $(n=20)$ and a model group $(n=40)$. After four weeks, the model group was subdivided into a model control group and a treatment group (Fuzheng Huayu capsule combined with Pegasys). The rats were administered a gavage of distilled water or a solution of the combined drugs [4.5 g Fuzheng Huayu (6 capsules) and $180 \mu \mathrm{g}$ Pegasys in $10 \mathrm{ml}$ distilled water] respectively, at a dosage of $10 \mathrm{ml} / \mathrm{kg}$, six times a week for four weeks. Blood was then taken from the inferior vena cava and the serum was obtained through centrifugation. In addition, liver tissues were prepared for further experiments.

The model group was intraperitoneally injected with $0.5 \%$ DMN ( $2 \mathrm{ml} / \mathrm{kg})$. The injections were administered on three successive days with two-thirds of a full dose and then no injections were administered for 4 days. This was continued for four weeks and the last two injections were performed with 
Table I. Number of apoptotic HSCs and hepatocytes and the expression of $\alpha$-SMA in the three groups of rats.

\begin{tabular}{lccc}
\hline Group & $\begin{array}{c}\text { Apoptotic HSCs } \\
\text { (per high power field, } \\
\text { magnification, } x \text { 40) }\end{array}$ & $\begin{array}{c}\text { Apoptotic hepatocytes } \\
\text { (per high power field, } \\
\text { (magnification, } \text { 40) }\end{array}$ & $\begin{array}{c}\alpha-S M A \\
\text { (percentage of positive area) }\end{array}$ \\
\hline Normal & 0 & $0.95 \pm 0.27$ & $1.46 \pm 0.13$ \\
Model & $0.15 \pm 0.05$ & $3.22 \pm 0.25$ & $8.42 \pm 0.53$ \\
Treatment & $0.27 \pm 0.08$ & $2.49 \pm 0.17$ & $4.11 \pm 0.45$ \\
F-value & 627.5 & 2.51 & 0.12 \\
P-value & $<0.05$ & $<0.05$ & $<0.05$ \\
\hline
\end{tabular}

Values are presented as the mean \pm standard deviation. HSCs, hemopoietic stem cells; SMA, smooth muscle actin.

one shot of two-thirds of a full dose and one shot of half of a full dose, respectively. An equal volume of saline was injected in the normal group. Once the DMN model was established, two rats were randomly sacrificed and the pathological condition of the liver was analyzed using an $\alpha$-SMA monoclonal antibody (Dako, Agilent Technologies, Santa Clara, CA, USA). The model was successfully established since liver fibrosis was observed. Following fixation of the liver tissues with $10 \%$ formalin, dehydration, transparency and wax immersion, cell apoptosis indices [apoptotic hemopoietic stem cell (HSC), apoptotic hepatocyte and $\alpha$-smooth muscle actin (SMA) levels] were analyzed using terminal deoxynucleotidyl transferase dUTP nick end labeling (TUNEL; Roche, Basel, Switzerland), $\alpha$-SMA and Masson triple staining (both from Shanghai Bogoo Biotechnology Co., Ltd., Shanghai, China). These experimental procedures were performed in accordance with the manufacturers' instructions.

Patients. A total of 100 patients with genotype 1 hepatitis $\mathrm{C}$ and hepatic cirrhosis from the Fifth People's Hospital of Wuxi (Wuxi, China) were analyzed. The patients were randomly divided into a treatment group (group A) and a control group (group B) with 50 patients in each group. In the treatment group, there were 28 males and 22 females, aged $42.3 \pm 10.2$ years old and with a disease course of $5.7 \pm 1.1$ years In the control group, there were 26 males and 24 females aged $40.3 \pm 3.4$ years old and with a disease course of $7.2 \pm 2.2$ years. All patients were HCV antibody positive, had an HCV RNA level of $\geq 500 \mathrm{cps} / \mathrm{ml}$ and an Ishak fibrosis score of 2, 3 or 4 (8). This study was performed in accordance with the Declaration of Helsinki and with approval from the Ethics Committee of the Fifth People's Hospital of Wuxi. Written informed consent was obtained from all participants.

Clinical trials. The two groups of patients were treated with $180 \mu \mathrm{g}$ Pegasys (Shanghai Roche Pharmaceuticals Co., Ltd, Shanghai, China) combined with 1,000-1,200 $\mu \mathrm{g}$ ribavirin for 48 weeks. The patients in group A were also treated with a $4.5 \mathrm{~g}$ Fuzheng Huayu capsule (Shanghai Yellow Sea Pharmaceutical Co., Ltd., Shanghai, China) each day. The patients in group B were given a placebo. The levels of aminotransferase (ALT), total bilirubin (TBiL), aspartate aminotransferase (AST), albumin, $\alpha$-fetoprotein (AFP) and total bile acids (TBA) were determined using a Beckman-Coulter 3XL flow cytometry instrument (Beckman-Coulter, Miami, FL, USA), a CEQ-8000 sequencer instrument (Beckman-Coulter, Miami, FL, USA), a PE-9600 PCR instrument (Perkin-Elmer, Fremont, CA, USA) and an 7600 automatic biochemistry analyzer (Hitachi Ltd., Tokyo, Japan). Liver fibrosis indicators, including serum hyaluronic acid (HA), laminin (LN), type IV collagen (IVC) and type III procollagen (PCIII) were detected using an ELISA kit (Euroimmun, Lübeck, Germany) and a TUNEL apoptosis kit (Roche, Basel, Swiss). Fas and FasL levels of PBMCs were detected with a Fas and FasL testing kit (Shenzhen Jingmei Biotech Co., Ltd., Shenzhen, China) according to the manufacturer's instructions.

Statistical analysis. The quantitative data was presented as the mean \pm standard deviation and analyzed using SPSS software, version 16.0 (SPSS Inc., Chicago, IL, USA). Statistical analysis was performed using a Student's t-test for comparison and a Spearman's rank correlation coefficient. $\mathrm{P}<0.05$ was considered to indicate a statistically significant difference.

\section{Results}

Animal results. The TUNEL, $\alpha-$ SMA and Masson staining results revealed that there were no apoptotic HSCs in the normal group. However, in DMN rats, a number of apoptotic HSCs were observed around the hepatic sinusoid. The number of apoptotic HSCs was greater in the DMN model rats than in the DMN group treated with Fuzheng Huayu capsule combined with Pegasys. A small number of hepatocytes became apoptotic in the normal group, while there was a significant increase in the number of apoptotic hepatocytes in the DMN group. The number of apoptotic cells was markedly reduced in the rats treated with Fuzheng Huayu capsule combined with that in the model rats (Table I).

Clinical observations. Significant differences in ALT, TBiL, AST, ALB, AFP and TBA were observed between the patients treated with Fuzheng Huayu capsule combined with Pegasys and those treated with Pegasys alone $(\mathrm{P}<0.01$; Table II). In addition, the levels of markers for hepatic fibrosis, including HA, LN, IVC and PCIII were significantly reduced in the combined treatment group $(\mathrm{P}<0.01$; Table III). These results 
Table II. Liver function following treatment with Fuzheng Huayu and Pegasys (treatment) or Pegasys alone (control).

\begin{tabular}{lcccccc}
\hline Group & ALT $(\mathrm{U} / \mathrm{l})$ & TBiL $(\mu \mathrm{mol} / \mathrm{l})$ & AST $(\mathrm{U} / \mathrm{l})$ & ALB $(\mathrm{G} / \mathrm{l})$ & AFP $(\mathrm{ng} / \mathrm{ml})$ & TBA $(\mu \mathrm{mol} / \mathrm{l})$ \\
\hline Control & $88.65 \pm 20.75$ & $37.85 \pm 9.65$ & $213.48 \pm 44.61$ & $25.29 \pm 1.65$ & $56.17 \pm 12.91$ & $52.89 \pm 21.61$ \\
Treatment & $33.21 \pm 14.65$ & $12.38 \pm 6.45$ & $65.26 \pm 21.47$ & $39.46 \pm 2.47$ & $19.23 \pm 5.63$ & $89.34 \pm 11.78$ \\
t-value & 4.30 & 2.78 & 4.51 & 5.12 & 8.56 & 4.24 \\
P-value & $<0.01$ & $<0.01$ & $<0.01$ & $<0.01$ & $<0.01$ & $<0.01$
\end{tabular}

Values are presented as the mean \pm standard deviation. ALT, aminotransferase; TBiL, total bilirubin; AST, aspartate aminotransferase; ALB, albumin; AFP, $\alpha$-fetoprotein; TBA, total bile acids.

Table III. Serum fibrosis indices following treatment with Fuzheng Huayu and Pegasys (treatment) or Pegasys alone (control).

\begin{tabular}{lcccc}
\hline Group & HA $(\mu \mathrm{g} / \mathrm{l})$ & $\mathrm{LN}(\mu \mathrm{g} / \mathrm{l})$ & IVC $(\mu \mathrm{g} / \mathrm{l})$ & PCIII $(\mu \mathrm{g} / \mathrm{l})$ \\
\hline Control & $287.04 \pm 67.27$ & $145.38 \pm 67.35$ & $135.19 \pm 68.79$ & $202.52 \pm 49.87$ \\
Treatment & $245.38 \pm 52.19$ & $122.73 \pm 71.19$ & $110.32 \pm 42.16$ & $177.27 \pm 72.26$ \\
t-value & 4.77 & 3.87 & 4.65 & 7.67 \\
P-value & $<0.05$ & $<0.05$ & $<0.05$ & $<0.05$ \\
\hline
\end{tabular}

Values are presented as the mean \pm standard deviation. HA, hyaluronic acid; LN, laminin; IVC, type IV collagen; PCIII, type III procollagen.

Table IV. Expression of Fas and FasL in the peripheral blood mononucleated cells of patients following treatment with Fuzheng Huayu and Pegasys (treatment) or with Pegasys alone (control).

\begin{tabular}{lcc}
\hline Group & Fas & FasL \\
\hline Control & $0.69 \pm 0.25$ & $0.82 \pm 0.17$ \\
Treatment & $0.42 \pm 0.15$ & $0.57 \pm 0.19$ \\
t-value & 6.43 & 4.78 \\
P-value & $<0.05$ & $<0.05$ \\
\hline
\end{tabular}

Values are presented as mean \pm standard deviation. FasL, Fas ligand.

indicate that Fuzheng Huayu capsule combined with Pegasys improves the efficacy of Pegasys in protecting the liver from fibrosis. The Fas and FasL levels in PBMCs were detected in the blood. The expression of Fas and FasL was attenuated following treatment with Fuzheng Huayu capsules (Table IV). The results demonstrate that the function of the immune system was influenced by liver fibrosis following treatment. This suggests that liver injury and hepatocyte apoptosis were significantly improved following treatment with Fuzheng Huayu capsule combined with Pegasys, as indicated by liver tissue pathology (Fig. 1).

\section{Discussion}

Chronic liver fibrosis is a common pathological process in various chronic liver diseases. If chronic hepatitis $\mathrm{C}$ is not treated effectively, it increases the risk of liver cirrhosis and
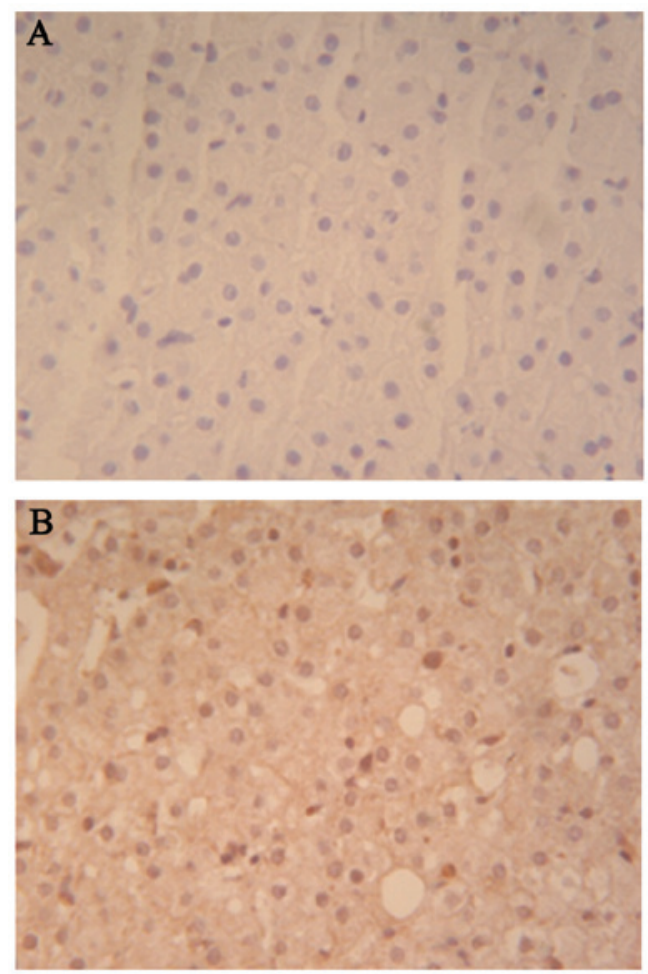

Figure 1. Liver tissue pathology following treatment with (A) Pegasys alone and (B) Fuzheng Huayu and Pegasys (magnification, $x$ 400). Hematoxylin-eosin staining was used.

cancer (9). The treatment and prevention of liver fibrosis has been investigated clinically. Fuzheng Huayu capsule has been shown to alleviate blood stasis and have a beneficial effect 
on the liver (10). In the present study, the effect of Fuzheng Huayu capsule combined with Pegasys was investigated by analyzing liver indicators following treatment.

Hepatocyte apoptosis was found to be significantly reduced in rats with DMN-induced liver injury following treatment with Fuzheng Huayu capsule combined with Pegasys. The decreased activation of HSCs, alleviation of endothelial cell damage and reduction of hepatocytes may be involved in this process. Reduced secretion of TGF- $\beta$ and TNF- $\alpha$, which promote the hepatocyte apoptosis, may be important for the function of these combined drugs $(11,12)$. These results demonstrated that Fuzheng Huayu capsule combined with Pegasys improved liver fibrosis induced by hepatitis $\mathrm{C}$, which are consistent with the previously reported studies (13-15). Fuzheng Huayu capsule includes: Salviae miltiorrhizae, which promotes blood circulation and alleviates blood stasis, as well as cooling and nourishing the blood; peach seed, which improves 'circulation of qi', alleviates blood stasis and activates enzymes required in bilirubin metabolism; Gynostemma pentaphyllum, which 'replenishes qi' to invigorate the spleen and regulates the immunity function; Cordyceps sinensis, which 'tonifies the spleen' and strengthens humoral immunity; and Schisandra, which 'supplements qi' and promotes the production of body fluid to increase the function of cell immunity $(16,17,18)$. The metabolism of $\mathrm{CCl}_{4}$ is also inhibited by Schisandra to protect hepatocytes (19). Based on this, Fuzheng Huayu capsule combined with Pegasys was used in the present study for the treatment of liver fibrosis, and it was hypothesized that the drug would improve the blood flow to the liver, strengthen liver detoxification and enhance hepatocyte regeneration.

In the present study, hepatocyte apoptosis was investigated in a DMN-induced rat model of liver fibrosis and clinical patients following treatment with Fuzheng Huayu capsule combined with Pegasys. The results demonstrate that there was a significant improvement in cell apoptosis following the treatment. The observed effects indicate that Fuzheng Huayu capsule and Pegasys efficiently enhance the body's immune function and promote liver regeneration (20-22). There are two aspects of liver fibrosis treatment: etiological treatment of the primary disease and treatment of liver fibrosis itself. The Fuzheng Huayu capsule combined with Pegasys treated the two aspects of the disease and decreased serum fibrosis indexes to inhibit or delay the development of liver fibrosis. This is the first study, to the best of our knowledge, to use the Fuzheng Huayu capsule combined with Pegasys for the treatment of patients with hepatitis $\mathrm{C}$ and hepatic cirrhosis. The clinical results demonstrate that the combined drugs exert certain curative effects in patients with hepatitis $C$ and increase their quality of life. In conclusion, the present study provides a method for the optimization of existing treatment strategies and for the establishment of potentially effective combination therapies.

\section{Acknowledgements}

This study was supported by the Social Development Project of Wuxi Administration of Science \& Technology (no. CSE01009).

\section{References}

1. Lavanchy D: The global burden of hepatitis C. Liver Int 29 (Suppl 1): 74-81, 2009.

2. Bota S, Sporea I, Popescu A, Sirli R, Neghina AM, Danila M and Strain M: Response to standard of care antiviral treatment in patients with HCV liver cirrhosis - a systematic review. J Gastrointestin Liver Dis 20: 293-298, 2011.

3. Michielsen P, Ho E and Francque S: Does antiviral therapy reduce the risk of hepatocellular carcinoma in patients with chronic hepatitis C? Minerva Gastroenterol Dietol 58: 65-79, 2012.

4. Ghany MG, Strader DB, Thomas DL and Seeff LB; American Association for the Study of Liver Diseases: Diagnosis, management, and treatment of hepatitis C: an update. Hepatology 49: 1335-1374, 2009.

5. Asian Pacific Association for the Study of the Liver (APASL) Hepatitis C Working Party; McCaughan GW, Omata M, Amarapurkar D, et al: Asian Pacific Association for the Study of the Liver consensus statements on the diagnosis, management and treatment of hepatitis $\mathrm{C}$ virus infection. J Gastroenterol Hepatol 22: 615-633, 2007.

6. Guedj H, Guedj J, Negro F, et al: The impact of fibrosis and steatosis on early viral kinetics in HCV genotype 1-infected patients treated with Peg-IFN-alfa-2a and ribavirin. J Viral Hepat 19: 488-496, 2012.

7. Heathcote EJ, Shiffman ML, Cooksley WG, et al: Peginterferon alfa-2a in patients with chronic hepatitis $\mathrm{C}$ and cirrhosis. N Engl J Med 343: 1673-1680, 2000.

8. Gordon SC: Treatment of viral hepatitis-2001. Ann Med 33: 385-390, 2001.

9. Wu JW and Wang W: The relationship of rat fibrosis and hyaluronic acid and related proteins. Chinese Journal of Anatomy 23: 268, 2010 (In Chinese).

10. Cui AL: Treatment of 54 cases of active hepatocirrhosis with lamivudine combined with $\mathrm{Fu}$ Zheng Hua Yu capsule. Journal of China Pharmaceutical 21: 74-75, 2012 (In Chinese).

11. De Bleser PJ, Niki T, Xu G, Rogiers V and Geerts A: Localization and cellular sources of activins in normal and fibrotic rat liver. Hepatology 26: 905-912, 1997.

12. Brenndörfer ED, Weiland M, Frelin L, et al: Anti-tumor necrosis factor $\alpha$ treatment promotes apoptosis and prevents liver regeneration in a transgenic mouse model of chronic hepatitis $\mathrm{C}$. Hepatology 52: 1553-1563, 2010.

13. Tan CY, Tan SZ, Jiang J and Xu LM: Effects of 'Fuzheng Huayu Decoction' on hepatocellular apoptosis in rats with DMN liver fibrosis. Shanghai Journal of Traditional Chinese Medicine 39: 47-49, 2005 (In Chinese).

14. Prati GM, Aghemo A, Rumi MG, et al: Hyporesponsiveness to PegIFNa2B plus ribavirin in patients with hepatitis C-related advanced fibrosis. J Hepatol 56: 341-347, 2012

15. Bzowej N, Nelson DR, Terrault NA, et al: PHOENIX: A randomized controlled trial of peginterferon alfa-2a plus ribavirin as a prophylactic treatment after liver transplantation for hepatitis C virus. Liver Transpl 17: 528-538, 2011.

16. Park $\mathrm{CH}$, Kim DH, Park MH, et al: Chinese prescription Kangen-karyu and Salviae Miltiorrhizae radix improve age-related oxidative stress and inflammatory response through the PI3K/Akt or MAPK pathways. Am J Chin Med 42: 987-1005, 2014.

17. Teng $\mathrm{H}$ and Lee WY: Antibacterial and antioxidant activities and chemical compositions of volatile oils extracted from Schisandra chinensis Baill. seeds using simultaneous distillation extraction method, and comparison with Soxhlet and microwave-assisted extraction. Biosci Biotechnol Biochem 78: 79-85, 2014.

18. Yu HY, Chen ZY, Sun B, et al: Lignans from the fruit of Schisandra glaucescens with antioxidant and neuroprotective properties. J Nat Prod 77: 1311-1320, 2014.

19. Cheng N, Ren N, Gao H, Lei X, Zheng J and Cao W: Antioxidant and hepatoprotective effects of Schisandra chinensis pollen extract on CCl4-induced acute liver damage in mice. Food Chem Toxicol 55: 234-240, 2013.

20. Li TF, Qin MZ and Hu SS: The expression of Fas and FasL in PBMC of multiple sclerosis patients. Shanghai Journal of Immunology 22: 131, 2002 (In Chinese).

21. Alkahtani S: Hepatitis C infection and apoptosis in hepatocellular carcinoma. Pak J Biol Sci 12: 804-808, 2009.

22. Zaki Mel S, Auf FA, Ghawalby NA and Saddal NM: Clinical significance of serum soluble Fas, Fas ligand and fas in intrahepatic lymphocytes in chronic hepatitis C. Immunol Invest 37: 163-170, 2008. 